\title{
On the tension between growth rate and CMB data
}

\author{
Alba Quelle ${ }^{\mathrm{a}}$, Antonio L. Maroto ${ }^{\mathrm{b}}$ \\ Departamento de Física Teórica and Instituto de Física de Partículas y del Cosmos (IPARCOS), Universidad Complutense de Madrid, \\ 28040 Madrid, Spain
}

Received: 17 December 2019 / Accepted: 15 April 2020 / Published online: 6 May 2020

(C) The Author(s) 2020

\begin{abstract}
We analyze the claimed tension between redshift space distorsions measurements of $f(z) \sigma_{8}(z)$ and the predictions of standard $\Lambda \mathrm{CDM}$ (Planck 2015 and 2018) cosmology. We consider a dataset consisting of 17 data points extending up to redshift $z=1.52$ and corrected for the Alcock-Paczynski effect. Thus, calculating the evolution of the growth factor in a $w \mathrm{CDM}$ cosmology, we find that the tension for the best fit parameters $w, \Omega_{m}$ and $\sigma_{8}$ with respect to the Planck $2018 \Lambda \mathrm{CDM}$ parameters is below $2 \sigma$ in all the marginalized confidence regions.
\end{abstract}

\section{Introduction}

Large-scale galaxy surveys are becoming one of the most powerful tools to test the currently accepted $\Lambda \mathrm{CDM}$ model based on General Relativity. The possibility of mapping the distribution of matter in large volumes at different redshifts allows to measure the growth rate of structures as a function of time and (length) scale which is a well-defined prediction of any cosmological model.

The ability of such surveys to construct 3D maps depends crucially on the precise determination of galaxy redshifts from which radial distances to the survey objects can be inferred. The actual conversion depends, in turn, on two important effects. On one hand, peculiar velocities introduce distorsions in the redshift distribution, the so called redshift space distorsions (RSD), generating an anisotropic galaxy power spectrum. On the other, although at low redshift the Hubble law provides a straightforward relation between redshift and distances, at higher redshifts this conversion depends on the chosen fiducial cosmology. This fact lays behind the Alcock-Paczynski (AP) effect. In recent times these effects have allowed to measure the linear growth rate of structures, defined as $f=d \ln \delta_{m} / d \ln a$ with $\delta_{m}$ the lin-

\footnotetext{
a e-mail: aquelle@ucm.es

be-mail: maroto@ucm.es (corresponding author)
}

ear matter density contrast with relatively good precision in a wide range of redshifts. More precisely, RSD provide a measurement of the quantity $f(z) \sigma_{8}(z)$, where $\sigma_{8}(z)$ is the normalization of the linear matter power spectrum at redshift $z$ on scales of $8 h^{-1} \mathrm{Mpc}$. In particular, measurements which can reach $10 \%$ precision have been obtained at $z<1$ by different surveys such as 2dF [1], 6dFGRS [2], WiggleZ [3] and recently by SDSS-III BOSS [4] and VIPERS [5]. At higher redshifts two measurements have also been obtained recently by FMOS [6] and from the BOSS quasar sample [7] although with relatively lower precision.

Confrontation of $f(z) \sigma_{8}(z)$ measurements with standard $\Lambda \mathrm{CDM}$ cosmology predictions has lead in recent years to claims of inconsistency or tension at different statistical significance levels. Thus in [8] a lower growth rate than expected from Planck $\Lambda \mathrm{CDM}$ cosmology was identified for the first time. Later on $[9,10]$ a tension at the $2 \sigma$ level was claimed between the Planck data and the CFHTLenS determination of $\sigma_{8}$. A similar tension was found by the KiDS+VIKING tomographic shear analysis [11]. More recently [12] a $3 \sigma$ tension with respect to the best fit parameters of Planck 2015 was also identified in a set of 18 data points from RSD measurements of $f(z) \sigma_{8}(z)$. The tension could even increase up to $5 \sigma$ if an extended dataset is used [13]. The extended dataset has far more data at low redshifts where the model discrimination is easier [14], however in this extended case possible correlations within data points have not been taken into account. ${ }^{1}$ The possibility that more recent datapoints with larger errorbars compared to earlier datapoints could introduce a bias towards the expected standard Planck/ $\Lambda \mathrm{CDM}$ cosmology is also discussed in [13].

In this paper, we follow a complementary approach, rather than using the extended dataset for which correlations are unknown, we will revisit the analysis of the tension from the most conservative point of view, i.e. using only independent datapoints or points whose correlations are known.

\footnotetext{
${ }^{1}$ See however [15] for a more recent analysis.
} 
Thus we consider the Gold dataset of [12] and introduce two changes in the analysis. Firstly, we include the most recent measurements from BOSS-Q [7] and on the other, we draw attention on the possible correlation between SDSSLRG and BOSS-LOWZ points. Notice that the former are obtained from the SDSS data release DR7 with practically the same footprint as the later, obtained from DR10 and DR11, but with less galaxies. In this sense, we explore the consequences of removing the two SDSS-LRG points from the analysis. In this sense, our dataset is very similar to that consider by Planck collaboration [16]. On the other hand, we will take the best fit parameters of Planck 2018 (CMB alone) given in [16] rather than Planck 2015 used in [12]. We consider the same type of $w \mathrm{CDM}$ cosmologies with three free parameters $\left(w, \Omega_{m}, \sigma_{8}\right)$ but obtain the confidence regions from the marginalized (rather than maximized) likelihoods. This enlarges the confidence regions so that the tension is found to be reduced below the $2 \sigma$ level for all the parameters combinations.

\section{Growth of structures and $f(z) \sigma_{8}(z)$}

Let us consider a flat Robertson-Walker background whose metric in conformal time reads

$d s^{2}=a^{2}(\eta)\left[-d \eta^{2}+\delta_{i j} d x^{i} d x^{j}\right]$

The evolution of matter density perturbations $\delta_{m}=$ $\delta \rho_{m} / \rho_{m}$ in a general cosmological model with non-clustering dark energy and standard conservation of matter is given for sub-Hubble scales by

$\delta_{m}^{\prime \prime}+\mathcal{H} \delta_{m}^{\prime}-\frac{3}{2} \mathcal{H}^{2} \Omega_{m}(a) \delta_{m}=0$

where prime denotes derivative with respect to conformal time, $\mathcal{H}=a^{\prime} / a$ and $\Omega_{m}(a)=\rho_{m} /\left(\rho_{m}+\rho_{D E}\right)$. In this work we will limit ourselves to $w \mathrm{CDM}$ cosmologies so that at late times

$\mathcal{H}^{2}=H_{0}^{2} a^{2}\left(\Omega_{m} a^{-3}+\left(1-\Omega_{m}\right) a^{-3(1+w)}\right)$

and

$\Omega_{m}(a)=\frac{\Omega_{m}}{\Omega_{m}+\left(1-\Omega_{m}\right) a^{-3 w}}$

The growth rate is defined as

$f=\frac{d \ln \delta_{m}}{d \ln a}$

which can be approximated by $f \simeq \Omega_{m}^{\gamma}(a)$ with $\gamma \simeq 0.55$ for $w \mathrm{CDM}$ models. Even though this fitting function provides accurate description for cosmologies close to $\Lambda \mathrm{CDM}$, since we are interested in exploring a wide range of parameter space, in this work we will obtain $f$ just by numerically solving (2) with initial conditions $\delta_{m}\left(a_{i}\right)=1$ and $\delta^{\prime}\left(a_{i}\right)=$ $1 / a_{i}$ with $a_{i}$ well inside the matter dominated era.

The matter power spectrum corresponding to the matter density contrast in Fourier space $\delta_{k}(z)$ with $1+z=1 / a$ is given by $P(k, z)=V\left|\delta_{k}(z)\right|^{2}$ with $V$ the volume. Thus the variance of the matter fluctuations on a scale $R$ is given by

$\sigma_{R}^{2}(z)=\frac{1}{2 \pi^{2}} \int P\left(k^{\prime}, z\right) W_{R}^{2}\left(k^{\prime}\right)^{2} d k^{\prime}$

with the window function defined as:

$W_{R}(k)=\frac{3}{k^{3} R^{3}}[\sin (k R)-k R \cos (k R)]$

Thus $\sigma_{8}(z)$ corresponds to $\sigma_{R}(z)$ at the scale $R=8 h^{-1} \mathrm{Mpc}$.

From the matter power spectrum it is possible to define the galaxy power spectrum as $P_{g}(k, z)=b^{2}(z) P(k, z)$ with $b(z)$ the bias factor.

From the observational point of view, galaxy surveys are able to determine the galaxy power spectrum in redshift space, which is given by

$$
\begin{aligned}
& P_{r, \mathrm{obs}}\left(k_{r}, \mu_{r} ; z\right) \\
& \quad=\frac{H(z) d_{A r}^{2}(z)}{H_{r}(z) d_{A}^{2}(z)} D^{2}(z) b^{2}(z)\left[1+\beta(z) \mu^{2}\right]^{2} P(k, z=0)
\end{aligned}
$$

where $H(z)=(1+z) \mathcal{H}(z)$,

$d_{A}(z)=\frac{1}{1+z} \int_{\frac{1}{1+z}}^{1} \frac{1}{a^{1 / 2}} \frac{d a}{H_{0} \sqrt{\Omega_{m}+\left(1-\Omega_{m}\right) a^{-3 w}}}$

is the angular diameter distance, $D(z)=\delta_{m}(z) / \delta_{m}(0)$ is the growth factor, $\beta(z)=f(z) / b(z)$ and $\mu$ is the cosine of the angle between $\hat{k}$ and the observation direction. Finally, the index $r$ denotes that the corresponding quantity is evaluated on the fiducial cosmology. Notice that the first factor in (8) corresponds to the AP effect, whereas the $\left(1+\beta \mu^{2}\right)^{2}$ factor is generated by the RSD. As we see RSD induce an angular dependence on the power spectrum which contains a monopole, quadrupole and hexadecapole contributions. From the measurements of monopole and quadropole it is possible to obtain the $f(z) \sigma_{8}(z)$ function that for simplicity in the following we will denote $f \sigma_{8}(z)$. The measured value depends on the fiducial cosmology, so that in order to translate from the fiducial cosmology used by the survey to other cosmology it is needed to rescale by a factor [12]

$\operatorname{ratio}(z)=\frac{H(z) d_{A}(z)}{H_{r}(z) d_{A, r}(z)}$

The fiducial cosmology correction could affect not only $f \sigma_{8}$ but also the power spectrum or even introduce additional multipoles in the galaxy power spectrum in redshift space. In principle, all these effects could be properly taken into account but, as shown in [13], in practice an approximated 
Table 1 Planck 2015 [17] and 2018 [16] (TT,TE,EE+lowE) best fit $\Lambda \mathrm{CDM}$ parameters

\begin{tabular}{lll}
\hline & Planck 2015 & Planck 2018 \\
\hline$\Omega_{m}$ & $0.3156 \pm 0.0091$ & $0.3166 \pm 0.0084$ \\
$w$ & -1 & -1 \\
$\sigma_{8}$ & $0.831 \pm 0.013$ & $0.8120 \pm 0.0073$ \\
\hline
\end{tabular}

approach is employed which relies on the introduction of correction factors. In our case, and in order to check the results of [12] and [13], we have chosen the same correction factors used in those references. The same factors were used in [8]. In any case, different approaches can change the significance of the tension.

\section{Testing Planck cosmology}

In order to confront the predictions of standard $\Lambda \mathrm{CDM}$ model with $f \sigma_{8}(z)$ measurements, we will obtain theoretical predictions for a general $w$ CDM model with three free parameters $\left(\Omega_{m}, w, \sigma_{8}\right)$ with $\sigma_{8}=\sigma_{8}(z=0)$. Our benchmark models will correspond to the Planck 2015 and Planck 2018 (TT,TE,EE+lowE) best fit parameters in Table 1.

On the other hand, our data points will correspond to measurements of SDSS [18-20]; 6dFGS [21]; IRAS [22,23]; 2MASS [22,24]; 2dFGRS [25], GAMA [26], BOSS [27], WiggleZ [28], Vipers [5], FastSound [6] and BOSS Q [7]. In Table 2 we show the 17 independent data points with the corresponding fiducial cosmology parameters corresponding to the so called Gold-2017 compilation of [12] which contains 18 robust and independent measurements based on galaxy or SNIa observations together with an additional independent BOSS quasar point. As mentioned before, we have removed the two SDSS-LRG-200 points since they are based on almost the same galaxy selection as the BOSS-LOWZ point from two heavily overlapping footprints with BOSSLOWZ including fainter galaxies. On the data provided by these surveys we will apply the fiducial cosmology correction given by (10).

Apart from the errors quoted in Table 2, the three points corresponding to WiggleZ are correlated. Thus the nondiagonal covariance matrix for the data points $11,12,13$ is given by:

$$
C_{i j}^{11,12,13}=10^{-3}\left(\begin{array}{ccc}
6.4000 & 2.570 & 0.000 \\
2.570 & 3.969 & 2.540 \\
0.000 & 2.540 & 5.184
\end{array}\right)
$$

Table 2 Data points from $[7,12]$

\begin{tabular}{lllll}
\hline Index & Dataset & $z$ & $f \sigma_{8}(z)$ & $\Omega_{m}$ \\
\hline 1 & 6dFGS+SnIa & 0.02 & $0.428 \pm 0.0465$ & 0.3 \\
2 & SnIa+IRAS & 0.02 & $0.398 \pm 0.065$ & 0.3 \\
3 & 2MASS & 0.02 & $0.314 \pm 0.048$ & 0.266 \\
4 & SDSS-veloc & 0.10 & $0.370 \pm 0.130$ & 0.3 \\
5 & SDSS-MGS & 0.15 & $0.490 \pm 0.145$ & 0.31 \\
6 & 2dFGRS & 0.17 & $0.510 \pm 0.060$ & 0.3 \\
7 & GAMA & 0.18 & $0.360 \pm 0.090$ & 0.27 \\
8 & GAMA & 0.38 & $0.440 \pm 0.060$ & 0.27 \\
9 & BOSS-LOWZ & 0.32 & $0.384 \pm 0.095$ & 0.274 \\
10 & SDSS-CMASS & 0.59 & $0.488 \pm 0.060$ & 0.307115 \\
11 & WiggleZ & 0.44 & $0.413 \pm 0.080$ & 0.27 \\
12 & WiggleZ & 0.60 & $0.390 \pm 0.063$ & 0.27 \\
13 & WiggleZ & 0.73 & $0.437 \pm 0.072$ & 0.27 \\
14 & Vipers PDR-2 & 0.60 & $0.550 \pm 0.120$ & 0.3 \\
15 & Vipers PDR-2 & 0.86 & $0.400 \pm 0.110$ & 0.3 \\
16 & FastSound & 1.40 & $0.482 \pm 0.116$ & 0.270 \\
17 & BOSS-Q & 1.52 & $0.426 \pm 0.077$ & 0.31 \\
\hline
\end{tabular}

and the total covariance matrix would be

$C_{i j}=\left(\begin{array}{cccc}\sigma_{1}^{2} & 0 & 0 & \ldots \\ 0 & C_{i j}^{11,12,13} & 0 & \ldots \\ 0 & 0 & \ldots & \sigma_{N}^{2}\end{array}\right)$

The corresponding $\chi^{2}$ is defined as

$\chi^{2}\left(\Omega_{m}, w, \sigma_{8}\right)=V^{i} C_{i j}^{-1} V^{j}$

with $V^{i}=f \sigma_{8, i}-\operatorname{ratio}\left(z_{i}\right) f \sigma_{8}\left(z_{i} ; \Omega_{m}, w, \sigma_{8}\right)$. Here $f \sigma_{8, i}$ corresponds to each of the data points in Table 2 and $f \sigma_{8}\left(z_{i} ; \Omega_{m}, w, \sigma_{8}\right)$ is the theoretical value for a given set of parameters values. In order to obtain the two-dimensional confidence regions for the different pairs of parameters, we will construct the marginalized likelihoods integrating the remaining parameter with a flat prior, ${ }^{2}$ i.e.

$L\left(w, \sigma_{8}\right)=N \int_{\Delta \Omega_{m}} e^{-\frac{1}{2} \chi^{2}\left(\Omega_{m}, w, \sigma_{8}\right)} d \Omega_{m}$

In particular for $\Omega_{m} \in[0.05 ; 0.9], w \in[-2.5 ; 0.5]$ and $\sigma_{8} \in[0.1 ; 4.0]$. We have checked that the confidence regions remain practically unchanged if we enlarge these intervals. Notice that this is one of the main differences with respect to [12] in which the remaining parameter was fixed to the Planck cosmology value. This procedure implies the introduction of a strong prior in the likelihood (14) from CMB data. However, if we want to determine the confidence regions obtained from $f \sigma_{8}$ data alone, no CMB information should be included in

2 The Mathematica code used for the numerical analysis presented in this work is available upon request from the authors. 


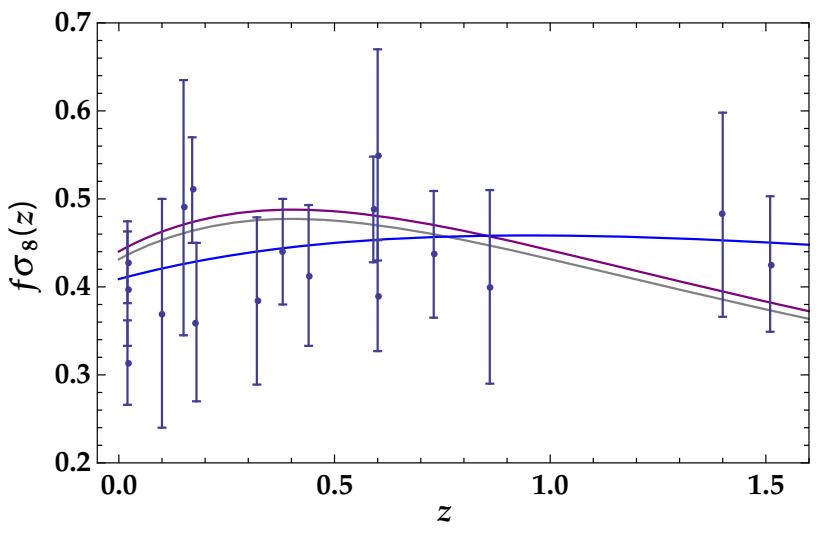

Fig. 1 Blue line corresponds to $w \mathrm{CDM}$ best fit, purple line corresponds to Planck 2015 and grey line to Planck 2018

Table 3 Values of $\chi^{2}$ for the models plotted in Fig. 1. $\chi_{L R G}^{2}$ refers to the dataset in [12] whereas $\chi_{N}^{2}$ refers to the data set in this work in Table 2. The best fit parameters for $w \mathrm{CDM}$ with $L R G$ data points are $\sigma_{8}=1.57, \Omega_{M}=0.1$ and $w=-0.27$. The tension between Planck cosmologies and $w \mathrm{CDM}$ for three fitted parameters is also shown in parenthesis

\begin{tabular}{lll}
\hline Model & $\chi_{L R G}^{2}$ & $\chi_{N}^{2}$ \\
\hline$w$ CDM & 16.51 & 10.09 \\
Planck 2015 & $21.58(1.38 \sigma)$ & $16.22(1.62 \sigma)$ \\
Planck 2018 & $18.32(0.51 \sigma)$ & $14.31(1.18 \sigma)$ \\
\hline
\end{tabular}

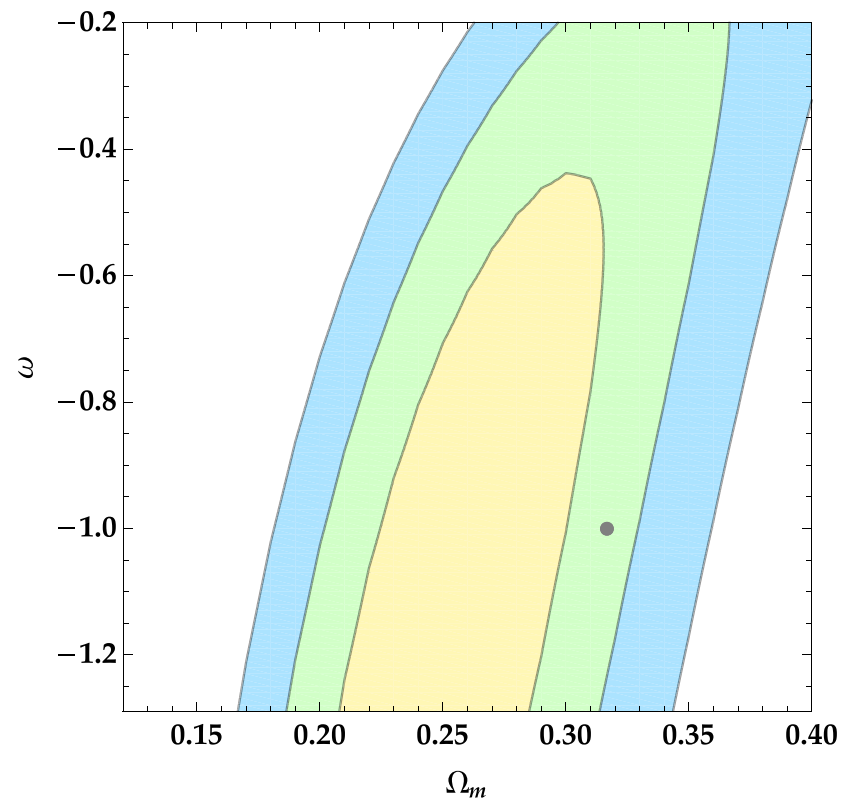

the corresponding likelihoods which is the approach considered in this work.

\section{Results}

In Fig. 1, the data points quoted in Table 2 together with the corresponding $w \mathrm{CDM}$ best fit curve are represented. The best fit corresponds to the parameters $\Omega_{m}=0.145, \sigma_{8}=$ 1.18 and $w=-0.46$. For the sake of comparison we also show the $f \sigma_{8}(z)$ curves corresponding to the Planck 2015 and Planck 2018 (in Table 1) cosmologies. The $\chi^{2}$ values for the different models can be found in Table 3 together with the corresponding tension level obtained from the $\chi^{2}$ difference for a three-parameter distribution. As we can see, for both models the tension of Planck cosmology with respect to the best fit $w$ CDM cosmology is below $2 \sigma$.

We see that Planck 2018 provides a better fit than the Planck 2015 cosmology, mainly thanks to the reduction in the $\sigma_{8}$ parameter, but still both are well above the best fit to $w \mathrm{CDM}$.

In order to obtain the corresponding confidence regions we will compare two procedures. On one hand, we will follow the approach in [12] in which the likelihood is maximized, i.e. in the two-dimensional confidence regions the remaining parameter is fixed to the corresponding Planck value in Table 1. In the second procedure, the remaining parameter is marginalized as mentioned in the previous section. In Figs. 2, 3 and 4 we show the different two-dimensional confidence

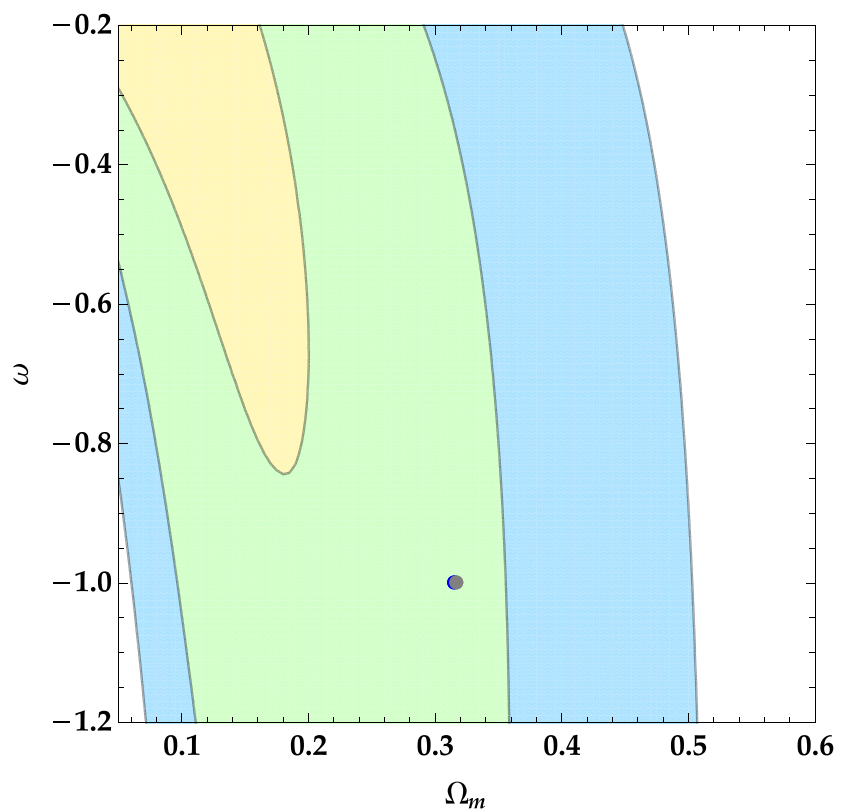

Fig. $2 w$ vs. $\Omega_{m} 1 \sigma, 2 \sigma$ and $3 \sigma$ confidence regions. Left: maximized contours with $\sigma_{8}=0.812$. The Planck 2018 point lays at $1.56 \sigma$. Right: marginalized contours. The blue point corresponds to Planck 2015 and lays at $1.71 \sigma$; and the grey point corresponds to Planck 2018 and lays also at $1.71 \sigma$ 

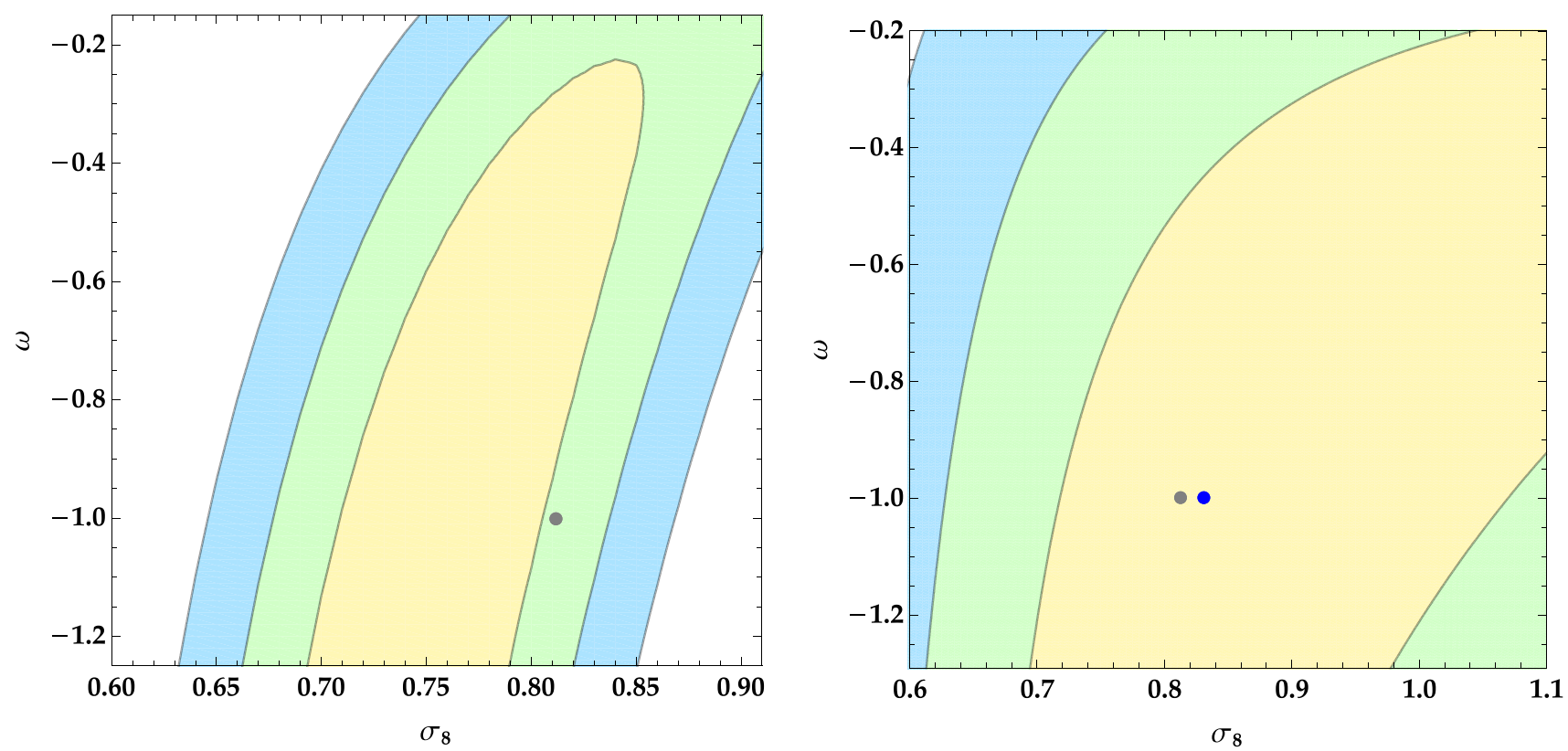

Fig. $3 w$ vs. $\sigma_{8} 1 \sigma, 2 \sigma$ and $3 \sigma$ confidence regions. Left: maximized contours with $\Omega_{m}=0.3166$. The Planck 2018 point lays at $1.19 \sigma$. Right: marginalized contours. The blue point corresponds to Planck 2015 and lays at $0.26 \sigma$ and the grey point corresponds to Planck 2018 and lays at $0.33 \sigma$
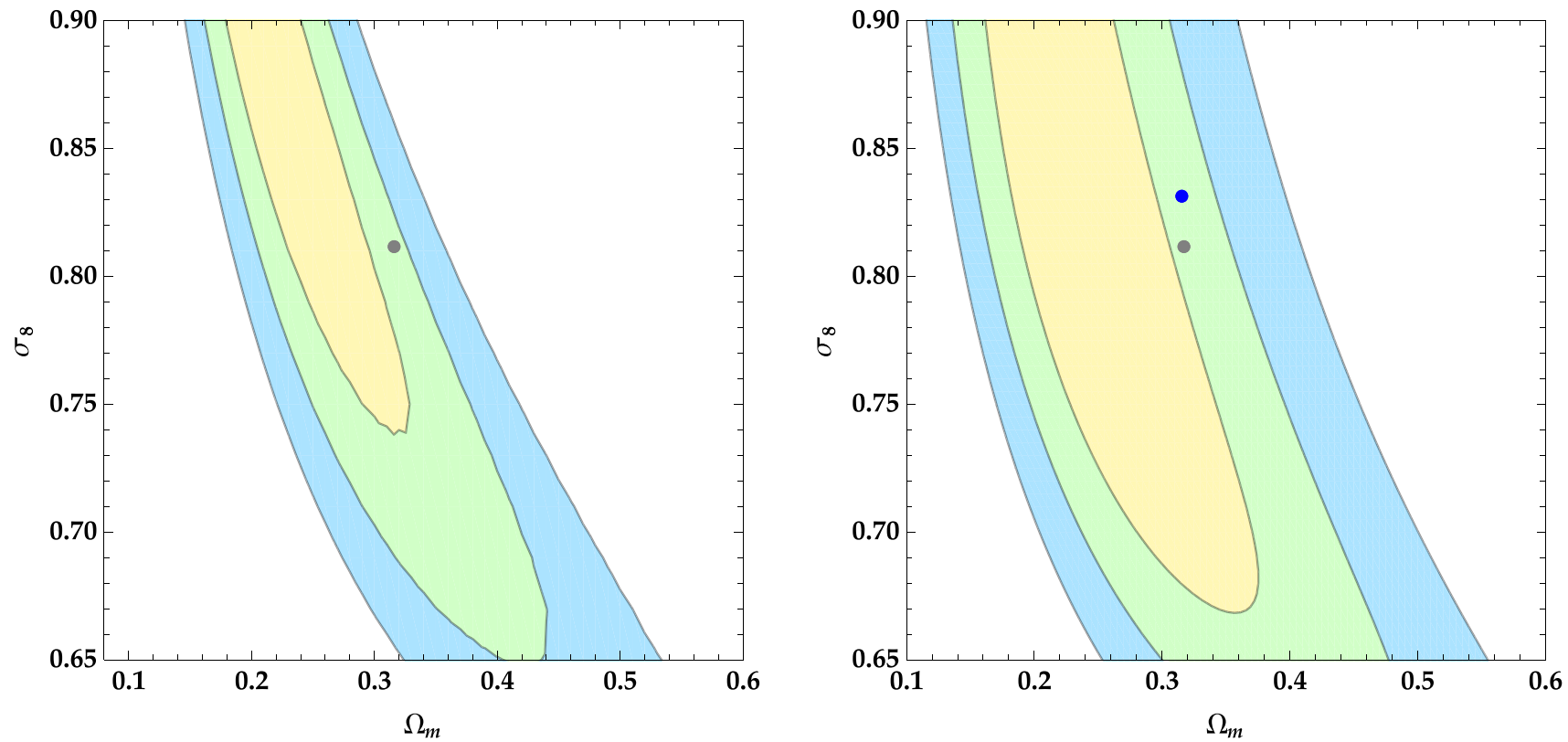

Fig. $4 \sigma_{8}$ vs. $\Omega_{m} 1 \sigma, 2 \sigma$ and $3 \sigma$ confidence regions. Left: maximized contours with $w=-1$. The Planck 2018 point lays at $1.68 \sigma$. Right: marginalized contours. The blue point corresponds to Planck 2015 and lays at $1.44 \sigma$. The grey point corresponds to Planck 2018 and lays at $1.23 \sigma$

contours. As we can see, Planck $2018 \Lambda \mathrm{CDM}$ shows tensions of $1.56 \sigma, 1.19 \sigma$ and $1.68 \sigma$ in the maximized contours which is around $1 \sigma$ below the tension found in [12] with Planck 2015 parameters, partially thanks to the reduced $\sigma_{8}$ value of Planck 2018 as mentioned before and the exclusion of the two SDSS-LRG points. On the other hand, the marginalized contours are as expected enlarged as compared to the maximized ones. Notice also that although the form of the $\left(\sigma_{8}, w\right)$ and $\left(\Omega_{m}, \sigma_{8}\right)$ contours are similar in both cases, the marginalization procedure changes the shape of the $\left(\Omega_{m}, w\right)$ regions and the tensions with respect to Planck 2018 are $1.71 \sigma$ for $\left(\Omega_{m}, w\right), 0.33 \sigma$ for $\left(\sigma_{8}, w\right)$ and in the $\left(\Omega_{m}, \sigma_{8}\right)$ plane we get $1.23 \sigma$. In Table 4 the different tension levels are summarized for the two Planck cosmologies, comparing maximized and 
Table 4 Tension levels for the Planck 2015 and Planck 2018 ACDM cosmologies in the different two-dimensional maximized and marginalized regions for the datasets in Table $2(N)$ and in [12] (LRG)

\begin{tabular}{llllll}
\hline & & $\operatorname{Max}_{L R G}$ & $\operatorname{Marg}_{L R G}$ & $\operatorname{Max}_{N}$ & $\operatorname{Marg}_{N}$ \\
\hline$\sigma_{8}-w$ & $\mathrm{P} 15$ & $2.70 \sigma$ & $0.29 \sigma$ & $1.76 \sigma$ & $0.26 \sigma$ \\
& $\mathrm{P} 18$ & $2.04 \sigma$ & $0.30 \sigma$ & $1.19 \sigma$ & $0.33 \sigma$ \\
$\Omega_{m}-w$ & $\mathrm{P} 15$ & $2.86 \sigma$ & $1.57 \sigma$ & $2.08 \sigma$ & $1.71 \sigma$ \\
& $\mathrm{P} 18$ & $2.22 \sigma$ & $1.58 \sigma$ & $1.56 \sigma$ & $1.71 \sigma$ \\
$\Omega_{m}-\sigma_{8}$ & $\mathrm{P} 15$ & $2.87 \sigma$ & $1.50 \sigma$ & $2.14 \sigma$ & $1.44 \sigma$ \\
& $\mathrm{P} 18$ & $2.25 \sigma$ & $1.28 \sigma$ & $1.68 \sigma$ & $1.23 \sigma$ \\
\hline
\end{tabular}

marginalized contours and with the dataset in this work and that in [12].

\section{Conclusions}

We have revisited the tension of $\Lambda \mathrm{CDM}$ Planck cosmology with RSD growth data. We have considered the Gold data set of [12] together with one additional BOSS-Q point and removing the two SDSS-LRG points thus obtaining a total of 17 independent data points.

Confronting these data with the growth rate obtained from a $w \mathrm{CDM}$ cosmology with three independent parameters $\left(w, \Omega_{m}, \sigma_{8}\right)$, we find that unlike previous claims, the tension with Planck 2018 cosmology is below the $2 \sigma$ level in all the two-dimensional marginalized confidence regions. This reduction, which is around $1.5 \sigma$ as compared to [12], is due to three different factors, namely, the use of Planck 2018 parameters, the fact that marginalized confidence regions have been considered and the exclusion of the possibly correlated SDSS-LRG points. Notice that for the $\Lambda$ CDM model (i.e. fixing $w=-1$ ), the tension is found to be at $1.68 \sigma$ level.

Future galaxy surveys such as J-PAS [29], DESI [30] or Euclid [31] with increased effective volumes will be able to reduce the error bars in the determination of $f \sigma_{8}(z)$ in almost an order of magnitude and will help to confirm or exclude the tension analyzed in this work.

Acknowledgements This work has been partially supported by MINECO grant FIS2016-78859-P(AEI/FEDER, UE) and by Red Consolider MultiDark FPA2017-90566-REDC.

Data Availability Statement This manuscript has no associated data or the data will not be deposited. [Authors' comment: All the data used in this work is already contained in the tables in our paper.]

Open Access This article is licensed under a Creative Commons Attribution 4.0 International License, which permits use, sharing, adaptation, distribution and reproduction in any medium or format, as long as you give appropriate credit to the original author(s) and the source, provide a link to the Creative Commons licence, and indicate if changes were made. The images or other third party material in this article are included in the article's Creative Commons licence, unless indicated otherwise in a credit line to the material. If material is not included in the article's Creative Commons licence and your intended use is not permitted by statutory regulation or exceeds the permitted use, you will need to obtain permission directly from the copyright holder. To view a copy of this licence, visit http://creativecomm ons.org/licenses/by/4.0/.

Funded by SCOAP 3 .

\section{References}

1. W.J. Percival et al., [2dFGRS Collaboration]. Mon. Not. R. Astron. Soc. 353, 1201 (2004). https://doi.org/10.1111/j.1365-2966.2004. 08146.x. arXiv:astro-ph/0406513

2. F. Beutler et al., Mon. Not. R. Astron. Soc. 423, 3430 (2012). https://doi.org/10.1111/j.1365-2966.2012.21136.x. arXiv:1204.4725 [astro-ph.CO]

3. C. Blake et al., Mon. Not. R. Astron. Soc. 415, 2876 (2011). https:// doi.org/10.1111/j.1365-2966.2011.18903.x. arXiv:1104.2948 [astro-ph.CO]

4. S. Alam et al. [BOSS Collaboration], Mon. Not. R. Astron. Soc. 470(3), 2617 (2017). https://doi.org/10.1093/mnras/stx721. arXiv:1607.03155 [astro-ph.CO]

5. A. Pezzotta et al., Astron. Astrophys. 604, A33 (2017). https://doi. org/10.1051/0004-6361/201630295. arXiv:1612.05645 [astroph.CO]

6. T. Okumura et al., Publ. Astron. Soc. Jpn. 68(3), 38 (2016). https:// doi.org/10.1093/pasj/psw029. arXiv:1511.08083 [astro-ph.CO]

7. P. Zarrouk et al., Mon. Not. R. Astron. Soc. 477(2), 1639 (2018). https://doi.org/10.1093/mnras/sty506. arXiv:1801.03062 [astroph.CO]

8. E. Macaulay, I.K. Wehus, H.K. Eriksen, Phys. Rev. Lett. 111(16), 161301 (2013). https://doi.org/10.1103/PhysRevLett.111.161301. arXiv:1303.6583 [astro-ph.CO]

9. N. MacCrann, J. Zuntz, S. Bridle, B. Jain, M.R. Becker, Mon. Not. R. Astron. Soc. 451(3), 2877 (2015). https://doi.org/10.1093/ mnras/stv1154. arXiv:1408.4742 [astro-ph.CO]

10. R.A. Battye, T. Charnock, A. Moss, Phys. Rev. D 91(10), 103508 (2015). https://doi.org/10.1103/PhysRevD.91.103508. arXiv:1409.2769 [astro-ph.CO]

11. H. Hildebrandt et al., arXiv:1812.06076 [astro-ph.CO]

12. S. Nesseris, G. Pantazis, L. Perivolaropoulos, Phys. Rev. D 96(2), 023542 (2017). https://doi.org/10.1103/PhysRevD.96. 023542. arXiv:1703.10538 [astro-ph.CO]

13. L. Kazantzidis, L. Perivolaropoulos, Phys. Rev. D 97(10), 103503 (2018). https://doi.org/10.1103/PhysRevD.97.103503. arXiv:1803.01337 [astro-ph.CO]

14. L. Kazantzidis, L. Perivolaropoulos, F. Skara, Phys. Rev. D 99(6), 063537 (2019). https://doi.org/10.1103/PhysRevD.99. 063537. arXiv:1812.05356 [astro-ph.CO]

15. F. Skara, L. Perivolaropoulos, arXiv:1911.10609 [astro-ph.CO]

16. N. Aghanim et al. [Planck Collaboration], arXiv:1807.06209 [astro-ph.CO]

17. P.A.R. Ade et al., [Planck Collaboration] Astron. Astrophys. 594, A13 (2016). https://doi.org/10.1051/0004-6361/201525830. arXiv:1502.01589 [astro-ph.CO]

18. L. Samushia, W.J. Percival, A. Raccanelli, Mon. Not. R. Astron. Soc. 420, 2102 (2012). https://doi.org/10.1111/j.1365-2966.2011. 20169.x. arXiv:1102.1014 [astro-ph.CO]

19. C. Howlett, A. Ross, L. Samushia, W. Percival, M. Manera, Mon. Not. R. Astron. Soc. 449(1), 848 (2015). https://doi.org/10.1093/ mnras/stu2693. arXiv:1409.3238 [astro-ph.CO]

20. M. Feix, A. Nusser, E. Branchini, Phys. Rev. Lett. 115(1), 011301 (2015). https://doi.org/10.1103/PhysRevLett.115.011301. arXiv:1503.05945 [astro-ph.CO] 
21. D. Huterer, D. Shafer, D. Scolnic, F. Schmidt, JCAP 1705(05), 015 (2017). https://doi.org/10.1088/1475-7516/2017/ 05/015. arXiv:1611.09862 [astro-ph.CO]

22. M.J. Hudson, S.J. Turnbull, Astrophys. J. 751, L30 (2013). https:// doi.org/10.1088/2041-8205/751/2/L30. arXiv:1203.4814 [astroph.CO]

23. S.J. Turnbull, M.J. Hudson, H.A. Feldman, M. Hicken, R.P. Kirshner, R. Watkins, Mon. Not. R. Astron. Soc. 420, 447 (2012). https://doi.org/10.1111/j.1365-2966.2011.20050.x. arXiv:1111.0631 [astro-ph.CO]

24. M. Davis, A. Nusser, K. Masters, C. Springob, J.P. Huchra, G. Lemson, Mon. Not. R. Astron. Soc. 413, 2906 (2011). https://doi. org/10.1111/j.1365-2966.2011.18362.x. arXiv:1011.3114 [astroph.CO]

25. Y.S. Song, W.J. Percival, JCAP 0910, 004 (2009). https://doi.org/ 10.1088/1475-7516/2009/10/004. arXiv:0807.0810 [astro-ph]
26. C. Blake et al., Mon. Not. R. Astron. Soc. 436, 3089 (2013). https:// doi.org/10.1093/mnras/stt1791. arXiv:1309.5556 [astro-ph.CO]

27. A.G. Sanchez et al., Mon. Not. R. Astron. Soc. 440(3), 2692 (2014). https://doi.org/10.1093/mnras/stu342. arXiv:1312.4854 [astro-ph.CO]

28. C. Blake et al., Mon. Not. R. Astron. Soc. 425, 405 (2012). https:// doi.org/10.1111/j.1365-2966.2012.21473.x. arXiv:1204.3674 [astro-ph.CO]

29. N. Benitez et al. [J-PAS Collaboration], arXiv:1403.5237 [astroph.CO]

30. A. Aghamousa et al. [DESI Collaboration], arXiv:1611.00036 [astro-ph.IM]

31. R. Laureijs et al. [EUCLID Collaboration], arXiv:1110.3193 [astro-ph.CO] 